\title{
Modelo de articulación para programas de educación superior frente a las pruebas estatales
}

\section{Articulation model for institutions programs of higher education facing state tests}

ROLDAN, Raúl F. ${ }^{1}$

RIAÑO, Diana²

AYALA, Elizabeth ${ }^{3}$

GÓMEZ, Carlos M. ${ }^{4}$

\begin{abstract}
Resumen
El sistema educativo es un proceso de formación permanente donde se propende por la formación de los estudiantes a partir de la educación inicial, hasta la educación superior. Proponemos un modelo de articulación de programas universitarios con respecto a las pruebas estatales para relacionar los saberes de los estudiantes con el conocimiento y las áreas en las cuales pretenden actuar. El modelo usa un enfoque sistémico para relacionar el uso de recursos, los propósitos emanados por los planes estratégicos y los valores que constituyen los sellos institucionales orientados hacia la misión y visión que persigue una organización.

Palabras clave: aseguramiento de la calidad; conocimiento y evaluación; pensamiento sistémico; excelencia académica
\end{abstract}

\begin{abstract}
The educational system is a permanent training process where students are trained from initial education to higher education. We propose a model for the articulation of university programs with respect to state tests to relate students' knowledge with knowledge and the areas in which they intend to act. The model uses a systemic approach to relate the use of resources, the purposes originating from the strategic plans and the values that constitute the institutional qualities oriented towards the mission and vision that an organization pursues.

key words: quality assurance; knowledge and evaluation; systemic thinking; academic excellence.
\end{abstract}

\section{Introducción}

El sistema educativo colombiano propende por la formación de los estudiantes a partir de la educación inicial, hasta la educación superior. Su propósito es garantizar la formación del estudiante durante su educación básica, que comprende cuatro niveles; la etapa de primera infancia, la primaria, la secundaria y la media, proceso que desarrolla el estudiante entre las edades de 0 a 15 años, seguido de la formación básica se encuentra el ciclo de educación superior, el cual se estructura de igual manera en 4 niveles; técnico, tecnológico, profesional y posgrado, sin embargo, para lograr vincular la educación básica y la educación profesional, se hace necesario

\footnotetext{
${ }^{1}$ Profesor e investigador, Facultad de Ciencias Empresariales. Fundación universitaria Compensar (Colombia)

2 Líder de Gestión de Contenidos. Fundación universitaria Compensar (Colombia)

${ }^{3}$ Directora de transversales, Departamentos de Ciencias Básicas y Sociohumanidades. Fundación universitaria Compensar (Colombia)

${ }^{4}$ Director de programa Diseño Visual, Facultad de Comunicación. Fundación universitaria Compensar (Colombia)
} 
desarrollar un proceso de evaluación continua, que establezca políticas y estrategias para lograr que el estudiante se proyecte hacia la realización de los todos los ciclos.

La definición del concepto de calidad en la educación superior, son múltiples, la (Unesco, 2010) la define así: "la calidad de la educación superior es un concepto multidimensional que debería comprender todas sus funciones y actividades: enseñanza y programas académicos, investigación y becas, dotación de personal, alumnos, infraestructura y entorno académico" (párr. 7); por su parte el Consejo de Acreditación - CNA - la define "a un atributo del servicio público de la educación en general y, en particular, al modo como ese servicio se presta, según el tipo de institución de que se trate" (CNA, 2019, párr. 1).

La estrategia diseñada por el Ministerio de Educación - MEN - para mejorar la calidad de la educación, intenta que todos los estudiantes, independiente de su procedencia, situación social, económica y cultural, cuenten con oportunidades para adquirir conocimientos, desarrollar las competencias y valores necesarios para convivir, ser productivos y seguir aprendiendo a lo largo de la vida. Para este propósito se crea el Sistema de Aseguramiento de la Calidad de la Educación Superior, encargado de velar por la articulación de los niveles educativos (inicial, preescolar, básica, media y superior). En la figura 1 se muestran los tres componentes del sistema que se relacionan entre sí para cumplir el objetivo de hacer seguimiento, desde diferentes instituciones, al proceso de educación en todos sus niveles.

Figura 1

Estructura del Sistema de Aseguramiento de la Calidad de la Educación

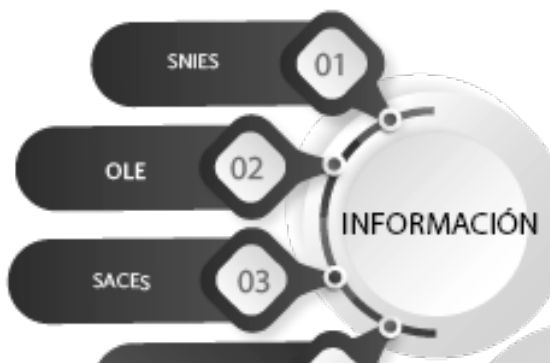

SPADIES

EVALUACIÓN

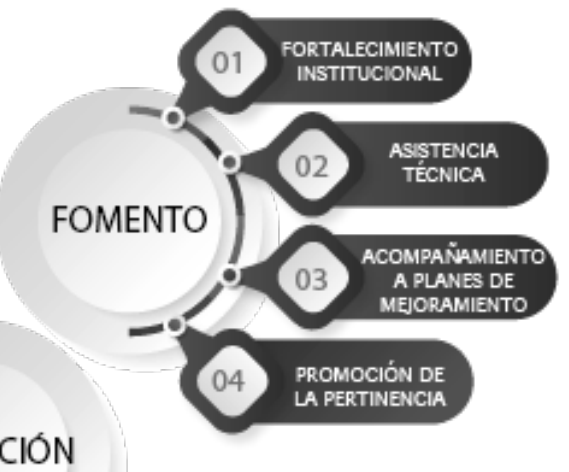

CESU

ICFES-SABER PRO (ECAES)

ICETEX

COLCIENCIAS

Fuente: Silva, Bernal y Hernández, 2014 
El sistema de información está destinado al impulso de los programas y la definición de políticas, el sistema de fomento ofrece asistencia técnica en procesos de acompañamiento a las instituciones que necesitan fortalecer sus métodos académicos, y el sistema de evaluación propende por el mejoramiento continuo de las instituciones, desde la actualización de los registros calificados hasta la búsqueda de la calidad del profesional egresado de cada institución, y el crecimiento que éste ha tenido en su proceso de aprendizaje. Para dar lugar al sistema de evaluación, el Instituto Colombiano para la Evaluación de la Educación (ICFES) realizan el seguimiento de la evolución del conocimiento en los diferentes niveles de educación, como se observa en la Figura 2, con el fin de identificar el nivel de desarrollo de competencias de un país, una región, las diferentes instituciones y el mismo estudiante.

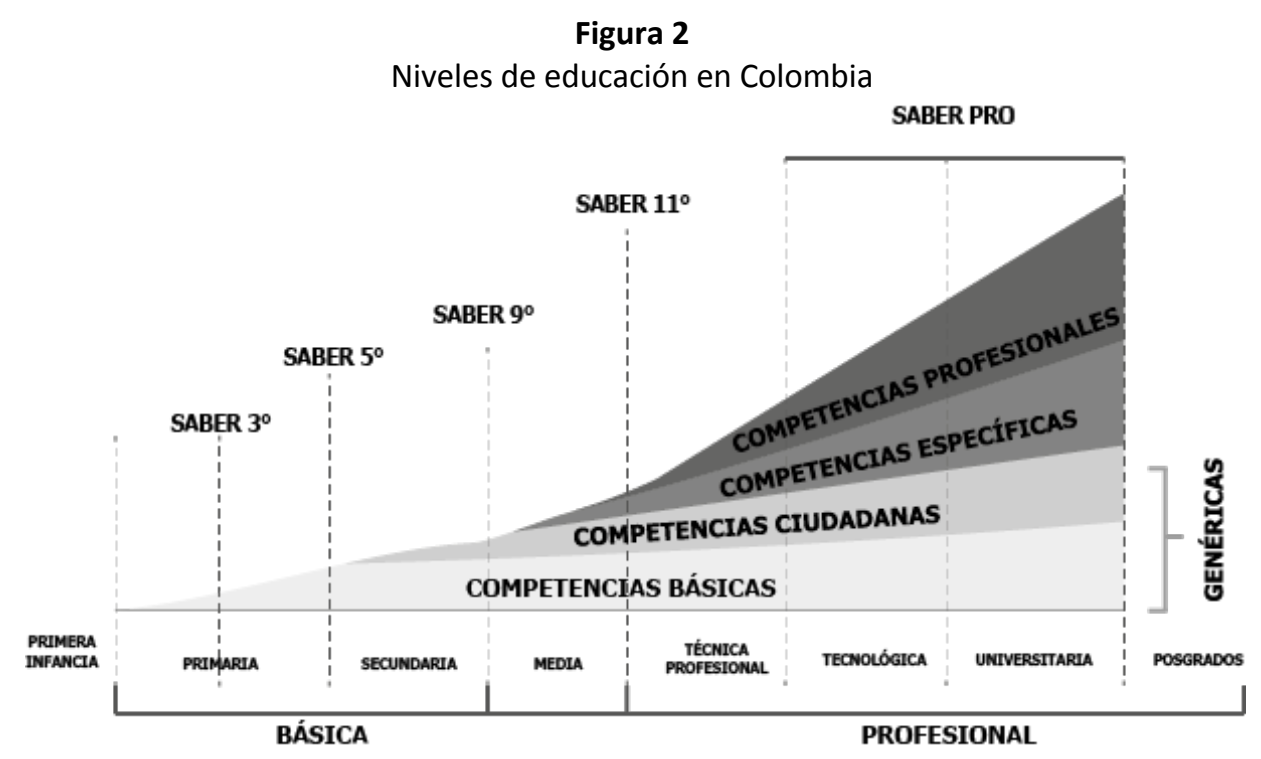

Fuente: Memorias taller uso de resultados examen Saber Pro-2019 (Pág. 115)

Para cumplir el propósito de evaluar el progreso de la educación desde los diferentes niveles, el ICFES propone la aplicación de las pruebas saber, que se implementa en diferentes niveles de la educación. Su objetivo principal es medir la influencia del sistema educativo en el estudiante a partir de las competencias que debería alcanzar en cada nivel. Las pruebas saber están estructuradas en módulos genéricas que permite al evaluador identificar competencias comunes en algunas áreas, y que mantienen su medición a lo largo de cada una de las pruebas saber; y módulos específicos que permiten al evaluador identificar el saber disciplinar en cada estudiante. Para la medición de los saberes el ICFES desarrolla las pruebas diseñadas y aplicadas bajo la metodología denominada diseño de especificaciones centrado en evidencias - DCE - esta metodología es "un marco para el desarrollo de pruebas planteado para hacer explícito lo que se mide en una evaluación y apoyar las inferencias hechas sobre el desempeño de los estudiantes con base en las evidencias derivadas de la prueba" (Magisterio, 2016); por lo tanto, para el caso de estudio en las Instituciones de Educación Superior -IES- , esta metodología exige trabajar en torno a un modelo de competencia enfocado en desarrollar habilidades y destrezas.

El desarrollo de la metodología se inició con la identificación de lo que se va a evaluar (conocimientos, competencias o habilidades), que en el caso de las pruebas saber se encuentran sustentados en los marcos de referencias de las evaluaciones. Una vez identificadas las conocimientos, habilidades o conocimientos a evaluar, la metodología lleva a formular las afirmaciones, que son enunciados que se hacen sobre los conocimientos, competencias o habilidades que se pretende inferir a partir de las respuestas dadas por los estudiantes en las pruebas. Desde cada afirmación se construyen las evidencias, es decir los productos observables que hacen posible verificar los desempeños a los que se refieren las afirmaciones. Las tareas son enunciados que dan pautas para la construcción de las preguntas o ítems de una prueba. En la figura 3, modelo usado actualmente en el diseño de pruebas en el ICFES se sintetiza el conjunto de procesos o pasos. 
Figura. 3

Modelo usado actualmente en el diseño de pruebas en el ICFES

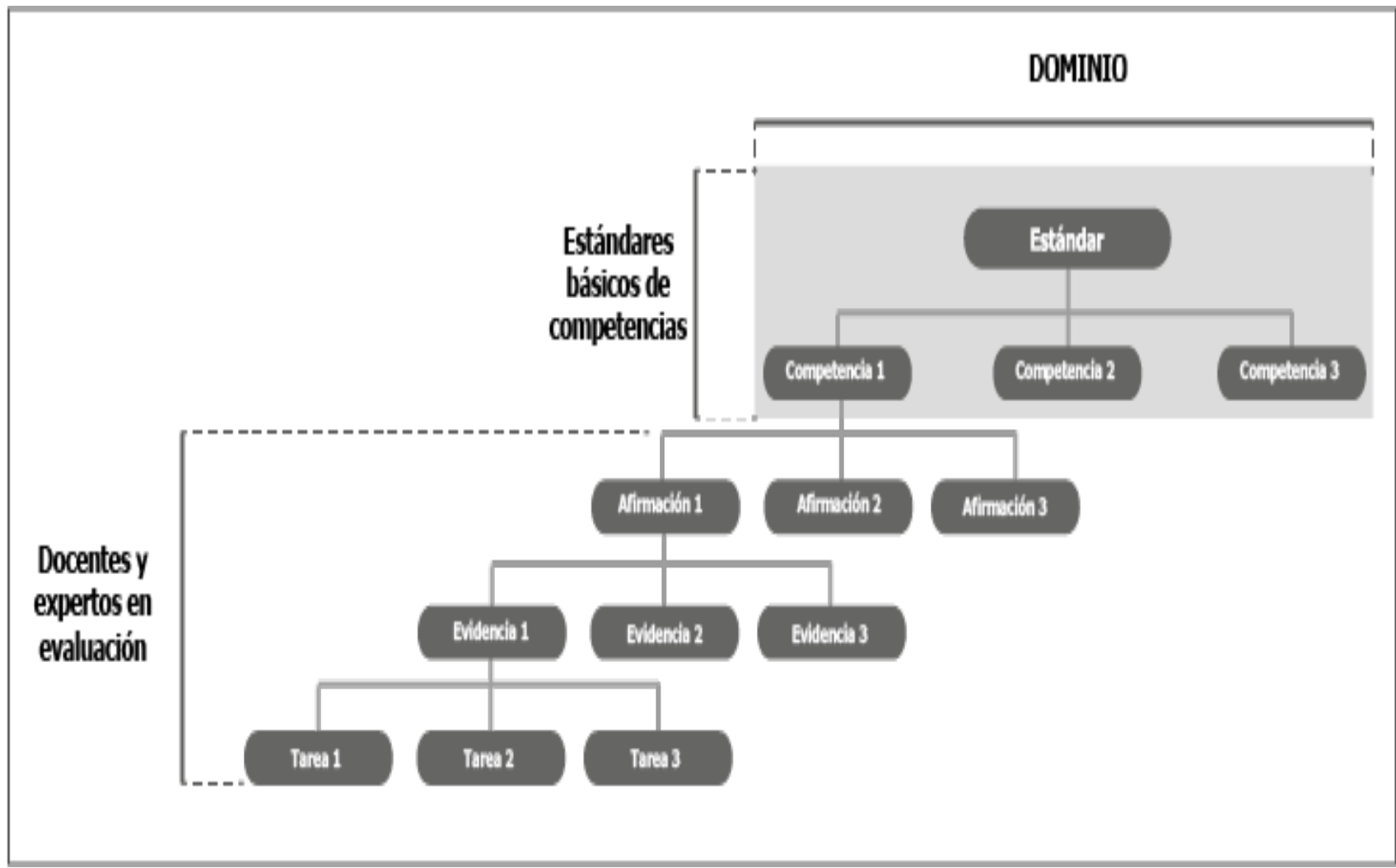

Fuente: https://www.icfes.gov.co/web/guest/acerca-del-examen-saber-pro

Sin embargo, para que una institución pueda comprender qué propone las pruebas saber, se hace necesario entender el concepto de competencias, el cual tiene múltiples definiciones haciendo complejo la comprensión del término. Según el Diccionario en línea de la Real Academia Española (DRAE 2018), la competencia se trata de un concepto polisémico, cuyo significado es "pericia, aptitud, idoneidad para hacer algo o intervenir en un asunto determinado" (pag. 2). Por ejemplo, el Proyecto Tunning define la competencia como "una combinación dinámica de atributos, en relación con procedimientos, habilidades, actitudes y responsabilidades, que describen los encargados del aprendizaje de un programa educativo o lo que los alumnos son capaces de demostrar al final de un proceso educativo" (Bravo, 2007, pág. 13). El Ministerio de Educación -MEN- considera la competencia como "conjunto de conocimientos, actitudes, disposiciones y habilidades (cognitivas, socioafectivas y comunicativas), relacionadas entre sí para facilitar el desempeño flexible y con sentido de una actividad en contextos relativamente nuevos y retadores. Por lo tanto, la competencia implica conocer, ser y saber hacer" (Trujillo, 2011, pág. 4), "el desempeño o la actuación integral de un sujeto, lo que implica conocimientos factúrales o declarativos, habilidades, destrezas, actitudes y valores, dentro de un contexto ético" (Pimienta, 2012, pág. 2), un "saber de ejecución", vinculada a un saber pensar, un saber desempeñar, un saber interpretar, así como un saber actuar en diversos escenarios. Se piensa entonces que una competencia moviliza diferentes capacidades, así como diversos contenidos en una situación real" (López, 2013, pág. 50). Tobón (2013) considera que la competencia "puede ser asumida como una actuación integral y razonada para hacer frente a la incertidumbre" (pág. 91), Oulett (2000) afirma, que también obedece a las actitudes y habilidades específicas de un individuo, definición que complementa Gómez (1997) incluyendo tres elementos determinantes, la intención, una acción, y un resultado; Bunk (1994) indica que el conocimiento esta correlacionado con las destrezas necesarias para el desarrollo de una acción, que según Bogoya (2000) debe estar enfocada a una situación concreta (Tobón 2013). Por lo tanto, se puede inferir que la competencia es un actuar que depende de un 
contexto especifico, de unos conocimientos previos, de la experiencia del individuo, de los valores que este aporta y de cómo se visiona la situación para dar solución a un problema.

Las competencias están definidas en función de los perfiles profesionales y es a través de las guías académicas o syllabus que se desglosan, asociando los resultados de aprendizaje (RA) y criterios de evaluación (CE) correspondientes. Los resultados del aprendizaje por su parte, "son declaraciones de lo que se espera que un estudiante conozca, comprenda y/o sea capaz de demostrar después de terminar un proceso de aprendizaje" (Aneca, 2013, pág. 15) citado en Tuning; el Marco Europeo de Cualificaciones lo define como "expresiones de lo que una persona en proceso de aprendizaje sabe, comprende y es capaz de hacer al culminar un proceso de aprendizaje" (Comisión Europea, 2009, pág. 3); y Pimienta como "formulaciones de lo que los estudiantes deben conocer, ser capaces de hacer o demostrar al concluir un trayecto formativo" (2012, pág.17). Por su parte los criterios de evaluación "en el ámbito pedagógico son las reglas objetivas que regirán el proceso de evaluación del aprendizaje, las que obviamente estarán teñidas como todo acto humano de cierta subjetividad" (Firgermann, 2014, pág. 1); "marco de referencia tomado por los docentes a la hora de evaluar el rendimiento académico de sus alumnos" (Definición.De, s.f., pág. 5)., en general es el marco de referencia como se mide el conocimiento adquirido.

Por lo tanto, si las pruebas saber pretenden la medición de competencias de la misma manera que las IES, debe existir un método que permita que los dos entes se encaminen hacia la misma dirección, siempre enfocados en el desarrollo del conocimiento del estudiante, de esta necesidad radica el desarrollo del modelo de las cinco fases, el cual propone este artículo. Para el desarrollo del presente proceso de investigación se realizó un estudio de caso con el programa de Ingeniería de Sistemas de una Institución de Educación Superior en la ciudad de Bogotá, Colombia.

El método empleado para alinear las visiones en el sistema de educación superior se basa en la cibernética, la cual estudia el comportamiento de los sistemas por medio de los ciclos de control en donde el concepto central es la variación en el comportamiento (Ashby, 2018), que está relacionada estrechamente en la teoría de control, las comunicaciones y la información (Arango y Pinzón, 2014), pero también se ha visto que a través del tiempo es usada para la descripción, diagnóstico y diseño de los sistemas de administración. Comúnmente se encuentra en la cibernética ciclos de primer y segundo nivel que pueden enfocarse hacia la administración; el primer nivel habla acerca del control del flujo de información y/o materiales y está orientado en la eficiencia, el segundo nivel implica el flujo de información hacia la alineación con los objetivos, lo que se relaciona con la planeación estratégica y se enfoca en la eficacia. En la cibernética de segundo orden se identifica el efecto que tiene el observador sobre el sistema observado (Yolles, 2006), así como los ciclos de control para los ciclos de primer orden. El tercer orden añade el ámbito en el que se mueve el sistema, es decir, la cultura, la filosofía y los valores entre otros, los cuales configuran los principios axiológicos en los que en sistema se enmarca y valida (Nova, Pinzón y Quintero, 2013).

\section{Metodología}

El objetivo que se persigue es articular la forma en que se evalúan los resultados de aprendizaje en los programas definidos, frente al modelo Saber Pro basado en desempeños.

\subsection{Población y muestra}

La población es la malla curricular donde se contempla la estructura del plan de estudios de un programa que se encuentra en curso y está vigente ante el MEN, denominado ingeniería de sistemas y está conformada por 65 asignaturas, de las cuales se toma una muestra del 13\%, correspondiente a 8 asignaturas específicas y transversales del currículo que tuviesen alguna relación con el módulo 36 "Formulación de proyectos de 
ingeniería". Es una investigación de enfoque cualitativo que permite hacer el análisis y la relación de la muestra con la afirmación 1 y 2 del módulo objeto de estudio de las pruebas Saber Pro.

\subsection{Modelo propuesto}

Los componentes de la cibernética de tercer orden tal y como se expresan anteriormente son descritos para disponer de un marco en la gestión, administración y el control de las actividades investigativas. En la Figura 4 se muestra la disposición de los nueve elementos que componen el modelo. En ésta los órdenes son identificados a partir de los ciclos de retroalimentación. En la figura 4, pueden observarse tres ciclos de retroalimentación: marco común, conocimiento y valor. Comenzando desde la parte superior de la Figura 4, en primera instancia, se mencionan los supuestos: el supuesto declarado y el supuesto usado. El supuesto declarado es lo que se dice que se va a hacer, para este caso es alcanzar la excelencia académica y el supuesto usado es lo que realmente se termina haciendo que es aplicar estrategias de evaluación y metodologías activas para el aprendizaje. El supuesto usado está conformado por un conjunto de premisas que fundamentan y permiten la operación - ejecución de los procedimientos. Generalmente existe una brecha entre lo que se dice que se va a hacer y lo que se termina haciendo, esto constituye una posibilidad de mejoramiento y de alineación de supuestos. Algunos tipos de investigación tienen por su naturaleza supuestos orientadores que determinan el rumbo de la investigación, por lo que son llamados "hipótesis" (Hurtado, 1998).

Figura 4

Modelo de articulación de programas universitarios con las pruebas de Estado empleando cibernética de tercer orden.

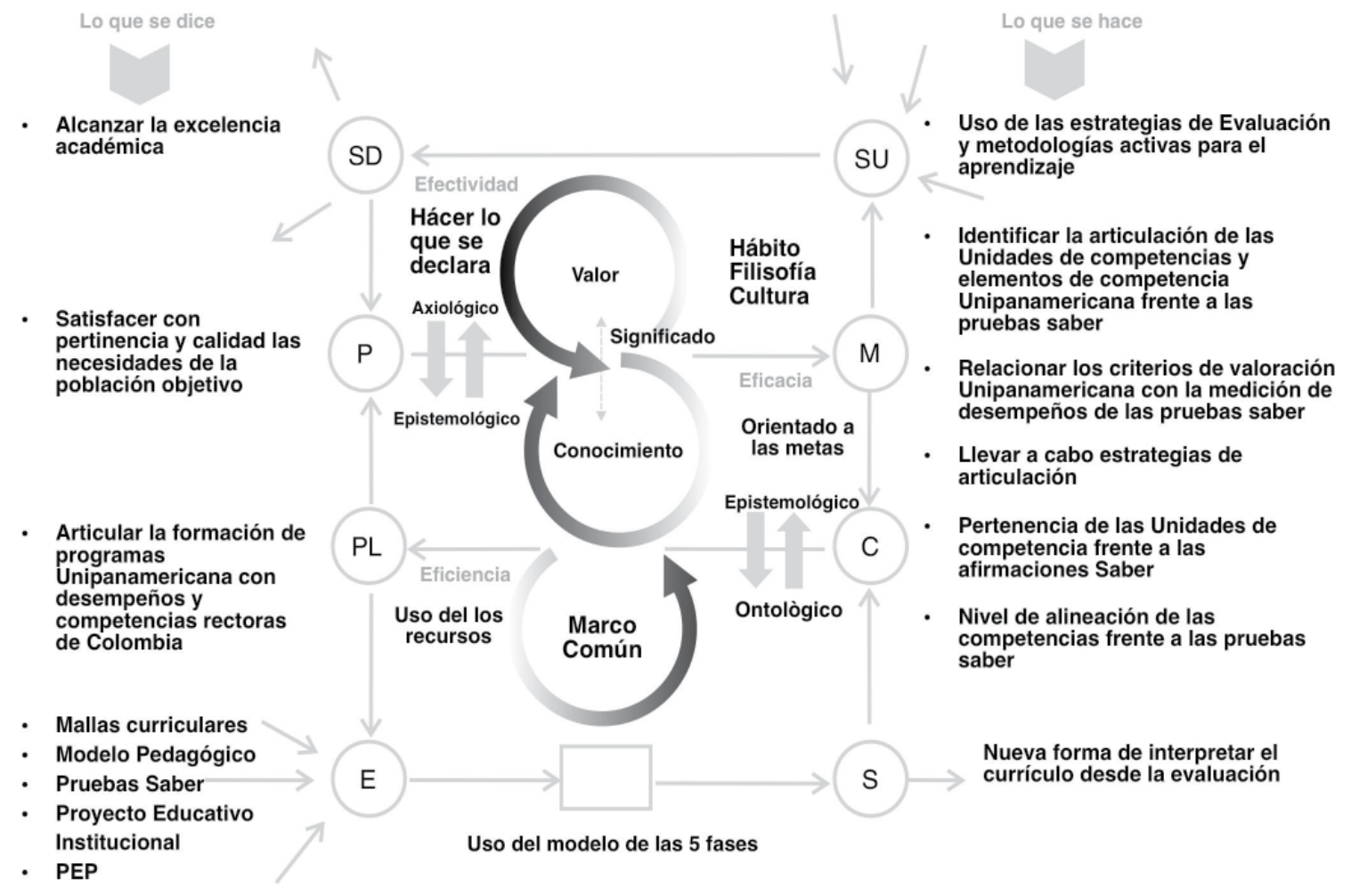

Fuente: los autores 
Desde arriba y hacia abajo en la figura 4, el siguiente elemento es el propósito que es satisfacer con pertinencia y calidad las necesidades de la población objetivo, este propósito está influenciado directamente por el investigador (el observador en un sistema). El investigador determina el fin último atribuido al proceso por el cual se acopla el sistema y se determina su sentido teleológico en cuanto a su explicación y a su comprensión (Páramo, 2017). El propósito se evidencia a través de las metas, las cuales son agrupaciones de actividades con propósitos, métodos, logros, y ámbitos definidos que desglosan y separan etapas interdependientes. Las metas están asociadas al cumplimiento del propósito dependiendo de las capacidades y habilidades del quienes realizan el proceso de investigación y las condiciones en las que se desarrolla. Las metas cambian en correspondencia a diferentes tipologías de investigación como lo plantea (Hurtado, 1998\}: exploratoria, descriptiva, comparativa, analítica, predictiva, proyectiva, interactiva, confirmatoria y evaluativa.

Un proceso de retroalimentación en un sistema tiene como fundamento el poder comparar la salida con la entrada, esta relación en el modelo de regulación propuesta comienza por medio del establecimiento de criterios de evaluación o juzgamiento. Un criterio es la regla que identifica el nivel de cumplimiento de las metas propuestas contra los resultados obtenidos. Frecuentemente estos elementos se describen junto con las metas y las salidas en el apartado rotulado como "discusión de los resultados de investigación". En (Páramo, 2017) se proponen tipos de criterios como los son: validez, utilidad, veracidad, pertinencia metodológica, credibilidad, plausibilidad y confiabilidad. La revisión de los criterios de la investigación pone de manifiesto una serie de diferencias entre los resultados y las metas, los cuales pueden ser traducidos en el plan. Un Plan es la descripción detallada de la estrategia que se sigue en la investigación para obtener lo que se desea. El plan determina que métodos y recursos son necesarios y en qué medida se requieren para el cumplimiento de las metas y los criterios previamente definidos. De esta forma se controla oportunamente los recursos para el desarrollo del proceso y de las actividades en correspondencia con las metas.

Todo proceso tiene asociado tres componentes: las Entradas, Transformación y Salida. Las Entradas consisten de una lista de cualidades, características, cantidades y adecuación de los tipos de recursos a ser usados dentro del proceso de investigación. Detallada en un nivel suficiente para ser utilizada en el proceso o transformación. La Transformación es la descripción del proceso ejecutado o de investigación que obedece a la estrategia utilizada en el plan. En investigación es conocida como metodología, aunque en esta etapa se ejecutan y se operacionaliza la estrategia. En ella, se identifica cómo los recursos de entrada permiten obtener los resultados o salidas, frecuentemente descrito en diferentes publicaciones bajo el encabezado de "métodos". Los métodos generalmente se clasifican en cualitativos y cuantitativos. Algunos ejemplos de estos métodos son los estudios de caso, grupos focales, grupos nominales, diagramas de afinidad, análisis documental, Delphi, historias de vida, o análisis de regresión, correlación canónica, series de tiempo, análisis factorial y análisis discriminante entre otros. Para realizar la transformación en el modelo propuesto, se emplea el modelo de las 5 fases. En cuanto a las Salidas son resultados del proceso de investigación, como valores estadísticos, conjuntos de datos, registros, que posteriormente se comparan contra los resultados buscados en las metas y los criterios propuestos. La salida esperada es una nueva forma de interpretar el micro currículo desde la evaluación.

En resumen, las Entradas, el Proceso, las salidas, los criterios y el plan, conforman lo que se conoce en la literatura como PHVA, (Moen y Norman, 2007) y (Johnson, 2002). El PHVA es el fundamento sobre el que se puede construtrir un ciclo de mejoramiento continuo y que son los pilares de la calidad (Buckler, 1996), (Bhuiyan y Baghel, 2005), (Gupta, 2006) y (Huarng, Horng y Chen, 1999). Desde la teoría de control se conoce como sistemas de control en lazo cerrado (Dorf y Bishop, 2011), (Kuo y Golnaraghi, 1995) y (d'Azzo y Houpis, 1995). En esencia son ciclos de mejora continua o ciclos de realimentación, que conduce a la eficiencia en el manejo de los recursos, los cuales son especialmente mencionados en las normas conducentes a la calidad, (Terziovski y Power, 2007). Así se constituye el primer orden de la regulación y control cibernética. El segundo orden, conduce a la eficacia del proceso investigativo al llevar el proceso hacia un propósito evidenciado en la meta. En el segundo orden los 
criterios, el plan, el propósito y las metas conforman un ciclo que permite que el proceso se dirija hacia su finalidad, hacia su sentido teleológico dirigido por el investigador. El tercer orden, lo conforman los supuestos, los cuales conducen a que el proceso investigativo se alinee en cuanto a lo que dice que va a hacer y lo que hace. Por lo tanto, este último orden conduce al proceso investigativo hacia la efectividad.

\subsection{Procedimiento de recogida y análisis de datos}

Primero se identificaron las asignaturas específicas y transversales del programa de Ingeniería de Sistemas y se contrastaron con el módulo objeto de estudio, teniendo en cuenta la malla curricular y la guía orientadora de pruebas saber Pro del ICFES como insumo de entrada. Para la selección y correlación del módulo con la malla curricular, se hizo una reunión de expertos con los docentes investigadores y estudiantes del programa, quienes, a partir de las experiencias vividas en el aula, sugirieron las asignaturas susceptibles de análisis. Luego se procedió a hacer el análisis mediante el método de las cinco fases, a partir de una herramienta diseñada en Excel que permitiera una fácil lectura del comparativo. Este formato consigna inicialmente las afirmaciones del módulo 36 en correlación a las asignaturas, los resultados de aprendizaje y los criterios de evaluación previamente identificados de la IES, pensado en determinar cómo se impacta el proceso de evaluación desde el micro currículo y a partir de los resultados de estas observaciones establecer estrategias que respondan al objetivo de la investigación que busca articular la forma en que se evalúan los resultados de aprendizaje en los programas definidos frente al modelo Saber Pro basado en un modelo de evidencias.

\section{Resultados}

El examen Saber Pro tiene dos sesiones asociadas a competencias genéricas y contenidos específicos. La primera sesión es obligatoria y se compone de 5 módulos que evalúan competencias genéricas (módulo de lectura crítica, módulo de razonamiento cuantitativo, módulo de competencias ciudadanas, módulo de comunicación escrita y módulo de inglés). En la segunda sesión, existen módulos asociados a temáticas y contenidos específicos, de acuerdo a su área de formación. Cada Institución de Educación Superior - IES -, tiene la opción de seleccionar una de las combinatorias ofertadas por el ICFES. Esta entidad ha dispuesto que la oferta de módulos específicos de los exámenes Saber Pro está definida por la combinación del Núcleo Básico del Conocimiento - NBC y el nivel de formación establecido para cada programa de acuerdo con la clasificación del Sistema Nacional de Información de la Educación Superior (SNIES), del Ministerio de Educación Nacional. En la Resolución 395 de 2018 se adoptan los grupos de referencia de los Exámenes del Estado Saber PRO y Saber Ty T a los cuales se hace referencia más adelante. El grupo de referencia para nuestro estudio de caso, ingeniería de sistemas quedo establecido de la siguiente manera: nivel de formación: Universitario; código de área:8; área de conocimiento: ingeniería, arquitectura, urbanismo y afines; código de área núcleo: 827; núcleo básico de conocimiento: ingeniería de sistemas, telemática y afines.

Una vez identificada y seleccionada el área de conocimiento -y el núcleo básico de conocimiento, se realiza el cruce con la tabla de combinatorias por NBC que publica el ICFES, y así de esta manera determinar el código de las combinatorias y los módulos asociados. En la tabla 1 se observa el código de la combinatoria y los módulos asociados para ingeniería de sistemas. 
Tabla 1

Combinatorias según la clasificación del núcleo básico de conocimientos para el programa Ingeniería de sistemas

\begin{tabular}{|c|c|c|c|c|c|c|}
\hline $\begin{array}{c}\text { Área de } \\
\text { conocimiento }\end{array}$ & $\begin{array}{l}\text { Núcleo básico } \\
\text { del } \\
\text { conocimiento- } \\
\text { NBC }\end{array}$ & Combinatorias & Módulo 0 & Módulo 1 & Módulo 2 & Módulo 3 \\
\hline \multirow{3}{*}{$\begin{array}{l}\text { *Ingeniería, } \\
\text { arquitectura, } \\
\text { urbanismo y } \\
\text { afines }\end{array}$} & $\begin{array}{l}\text { Ingeniería de } \\
\text { sistemas, } \\
\text { telemática y } \\
\text { afines }\end{array}$ & 49 & Genéricas & $\begin{array}{l}\text { Formulación de } \\
\text { proyectos de } \\
\text { ingeniería }\end{array}$ & $\begin{array}{l}\text { Diseño de } \\
\text { software }\end{array}$ & $\begin{array}{l}\text { Pensamiento } \\
\text { científico } \\
\text { matemáticas y } \\
\text { estadística }\end{array}$ \\
\hline & $\begin{array}{l}\text { Ingeniería de } \\
\text { sistemas, } \\
\text { telemática y } \\
\text { afines }\end{array}$ & 36 & Genéricas & $\begin{array}{l}\text { Formulación de } \\
\text { proyectos de } \\
\text { ingeniería }\end{array}$ & $\begin{array}{l}\text { Pensamiento } \\
\text { científico } \\
\text { matemáticas y } \\
\text { estadística }\end{array}$ & \\
\hline & $\begin{array}{l}\text { Ingeniería de } \\
\text { sistemas, } \\
\text { telemática y } \\
\text { afines }\end{array}$ & 0 & Genéricas & \multicolumn{3}{|c|}{$\begin{array}{l}\text { Quienes tomen esta combinatoria NO presentan } \\
\text { módulos específicos, únicamente presentan los } \\
\text { módulos genéricos de la primera sesión. }\end{array}$} \\
\hline
\end{tabular}

* El área de conocimiento es amplia y además de aplicarse a ingeniería tiene ámbito en otras áreas según establece la norma

Fuente: Combinatoria NBC - ICFES

La combinatoria seleccionada por ingeniería de sistemas fue la 36, que consta de dos módulos, pero para el estudio de caso se trabajó con el módulo 1, formulación de proyectos de ingeniería. Las pruebas de estado en los módulos específicos fueron elaboradas a partir de documentos conceptuales propios de cada especialidad, que sirven de guía a la comunidad educativa en general y están interesados en conocer los pormenores del mismo, con la información básica referente a: la competencia evaluada, las afirmaciones y evidencias que componen la competencia. La competencia está asociada a evaluar las capacidades del estudiante para resolver problemas de su entorno, cada afirmación asocia el aprendizaje alcanzado y es medido desde los desempeños a través de una serie de evidencias que son descritas en el módulo.

\subsection{Aplicación del modelo de las cinco fases}

Mariano M. (2003) menciona que el término modelo, representa un patrón a seguir o un comportamiento similar que se quiere imitar para comprender la teoría que se emplea y explicar algo. Esto implica enlazar la parte teórica del proceso con la práctica en la vida real, a fin de adquirir una mayor comprensión y puesta en escenario, aquello que se puede considerar como abstracto. El modelo de las cinco fases propuesto es parte del primer ciclo expuesto en el marco metodológico, denominado ontológico y se presenta para la esquematización de un sistema de articulación entre los módulos específicos que evalúan las pruebas de estado de la educación superior (ES) y el micro currículo del programa. En este sistema de articulación se relaciona el micro currículo con las afirmaciones y evidencias de los módulos. Es así como los módulos específicos de las pruebas de estado ES para el nivel profesional Saber PRO y las guías académicas, son los insumos del modelo de las 5 fases (Figura 5). 


\section{Figura 5}

Diagrama del modelo de las 5 fases

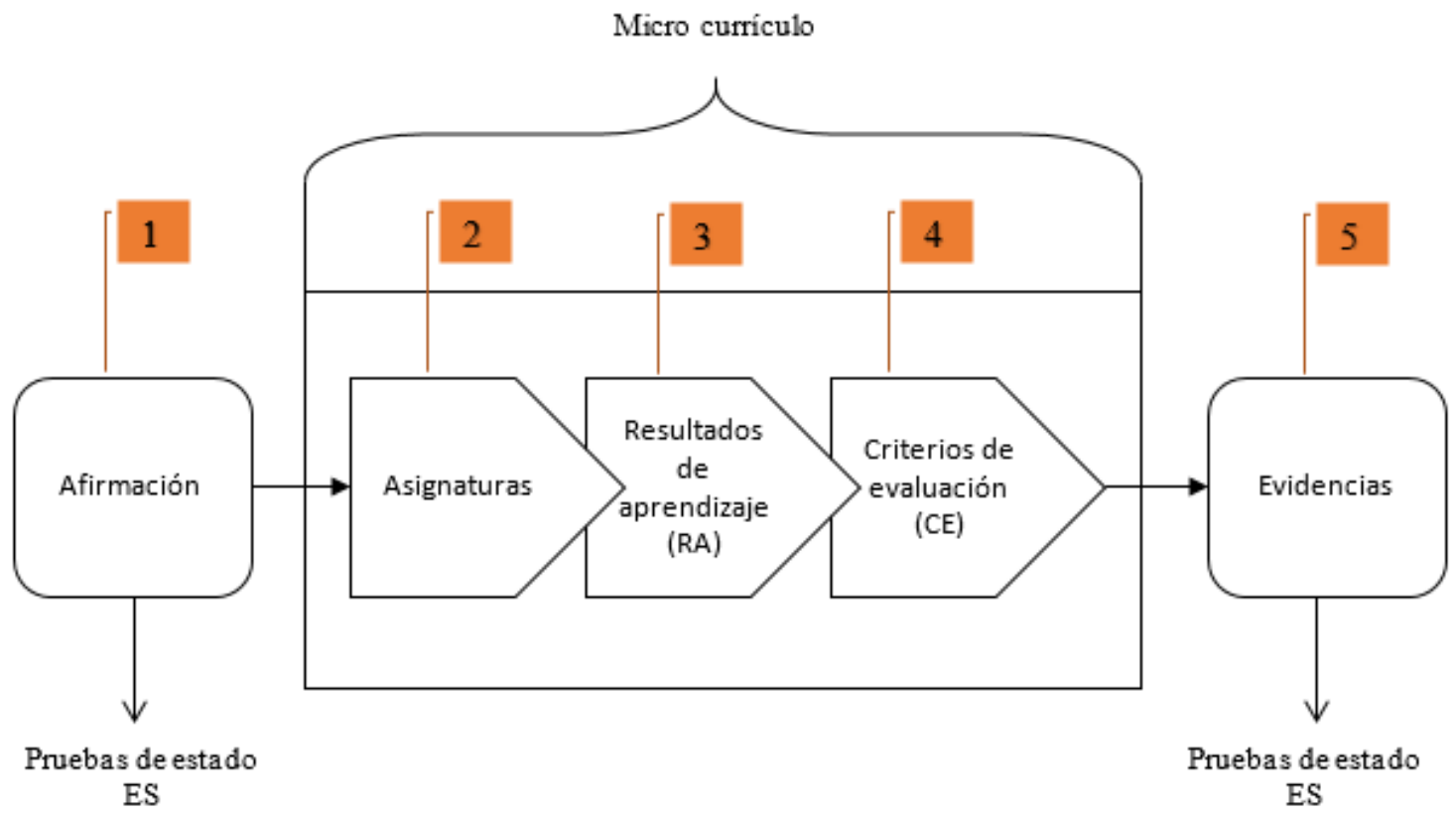

La fase 1 y 5 son la entrada y salida del sistema y la materia prima son los módulos específicos de las pruebas de estado saber PRO. La fase 2, 3 y 4 hacen parte del sistema de procesamiento central, donde se hace el análisis detallado de la guía académica institucional, a partir de los resultados de aprendizaje y criterios de evaluación, contemplados para lograr las competencias del programa académico. En fase 1, se identifican las afirmaciones de los módulos específicos de las pruebas de estado que, según el NBC, le corresponde previa selección de combinatoria por parte del programa o institución. En fase 2, se asocian las asignaturas del plan de estudios vigente, identificadas como claves en el fortalecimiento de los desempeños del módulo objeto de estudio, vista desde algún aspecto. En fase 3, se relacionan los resultados de aprendizaje que desde las asignaturas asociadas le impactan al módulo y en particular a las afirmaciones descritas en fase 1 . En fase 4, los criterios de evaluación que corresponden a cada resultado de aprendizaje son seleccionados meticulosamente, de modo que sean afines a la afirmación analizada. La fase 5, determina si los criterios de evaluación seleccionados se encuentran directa o indirectamente relacionados con las evidencias descritas.

Para aplicar el modelo, primero se identificaron las afirmaciones y evidencias del módulo a estudiar, que para el caso fue "Formulación de proyectos de ingeniería", cómo se observa en la Tabla 3 y las asignaturas relacionadas con el módulo: a. Gerencia y gestión de proyectos. b. Metodologías para el manejo de información. c. Emprendimiento, d. Economía para ingenieros, e. Teoría y modelos organizacionales. 
Tabla 3

Relación de afirmaciones y evidencias del

módulo Formulación de proyectos de ingeniería

\begin{tabular}{|c|c|}
\hline Afirmación (A) & Evidencia (E) \\
\hline $\begin{array}{l}\text { A1- Reconoce e identifica } \\
\text { condiciones políticas, legislativas, } \\
\text { socioeconómicas, técnicas y } \\
\text { ambientales del entorno, relevantes } \\
\text { para la caracterización y formulación } \\
\text { de proyectos. }\end{array}$ & $\begin{array}{l}\text { 1.1 Caracteriza el proyecto de acuerdo con su índole o naturaleza social, } \\
\text { económica, de inversión (entre otros) y el entorno utilizando referentes } \\
\text { apropiados } \\
\text { 1.2 Aplica las metodologías apropiadas para la formulación de un proyecto }\end{array}$ \\
\hline $\begin{array}{l}\text { A2- Formula y evalúa el proyecto, } \\
\text { apoyándose en un marco } \\
\text { metodológico pertinente, a partir de } \\
\text { las consideraciones del entorno y del } \\
\text { análisis de alternativas. }\end{array}$ & $\begin{array}{l}\text { 1.3 Formula el proyecto, apoyándose en un marco metodológico pertinente, a } \\
\text { partir de las consideraciones del entorno y del análisis de alternativas. } \\
\text { 1.4 Analiza e interpreta la viabilidad financiera de un proyecto. } \\
\text { 1.5 Cuantifica, en el marco de la planeación de un proyecto, elementos } \\
\text { fundamentales como alcance, tiempo y costo }\end{array}$ \\
\hline $\begin{array}{l}\text { A3- Reconoce su papel y } \\
\text { responsabilidad disciplinar, social y } \\
\text { ética como ingeniero en un contexto } \\
\text { de desempeño profesional. }\end{array}$ & $\begin{array}{l}\text { 1.6 Identifica sus responsabilidades sociales y técnicas en el ejercicio de su } \\
\text { profesión frente a referentes de actuación como códigos y normas. } \\
\text { 1.7 Asume una posición ética ante una situación en el desarrollo de un proyecto } \\
\text { de ingeniería. }\end{array}$ \\
\hline
\end{tabular}

Se realizó el cruce con las cinco asignaturas seleccionadas y con las tres afirmaciones del módulo formulación de proyectos de ingeniería. Para el presente documento se explicará el modelo con la afirmación 1, "reconoce e identifica las condiciones políticas, legislativas, socioeconómicas, técnicas y ambientales del entorno, relevantes para la caracterización y formulación de proyectos" y la asignatura Gerencia y Gestión de Proyectos. En la tabla 4 se observan los resultados de aprendizaje y criterios de evaluación de Gerencia y Gestión de Proyectos.

Tabla 4

Resultados de aprendizaje y criterios de evaluación de Gerencia y Gestión de Proyectos

\begin{tabular}{|c|c|}
\hline \multicolumn{2}{|r|}{ Asignatura: Gerencia y gestión de proyectos } \\
\hline Resultados de aprendizaje & Criterios de evaluación \\
\hline \multirow{3}{*}{$\begin{array}{l}\text { RA1. Distinguir las características y } \\
\text { elementos que componen la gestión } \\
\text { de un proyecto. }\end{array}$} & CE1. Identifica los fundamentos y las características de la gestión de proyectos. \\
\hline & $\begin{array}{l}\text { CE2. Identifica los aspectos y componentes presentes en la formulación de un } \\
\text { proyecto. }\end{array}$ \\
\hline & CE3. Conoce el proceso de implementación de un proyecto. \\
\hline \multirow{3}{*}{$\begin{array}{l}\text { RA2. Aplicar competenciaslaborales y } \\
\text { personales para la formulación, } \\
\text { implementación y evaluación de } \\
\text { proyectos, así como para el eficaz } \\
\text { funcionamiento de los ambientes de } \\
\text { trabajo. }\end{array}$} & CE4. Establece las fuentes de ideas de proyectos. \\
\hline & CE5. Evalúa las fuentes de ideas de proyectos. \\
\hline & $\begin{array}{l}\text { CE6. Distingue las características esenciales de la evaluación de un proyecto de } \\
\text { gestión. }\end{array}$ \\
\hline \multirow{2}{*}{$\begin{array}{l}\text { RA3. Explorar y valorar la importancia } \\
\text { de la gestión de proyectos para el } \\
\text { desarrollo profesional y de las } \\
\text { organizaciones. }\end{array}$} & $\begin{array}{l}\text { CE7. Define su propio proyecto a partir de la identificación de una idea de } \\
\text { proyecto. }\end{array}$ \\
\hline & $\begin{array}{l}\text { CE8. Gestiona un proyecto dentro de su entorno laboral inmediato, a partir de la } \\
\text { apropiación de la temática de la asignatura. }\end{array}$ \\
\hline
\end{tabular}

Los criterios de evaluación que pertenecen a los resultados de aprendizaje, fueron comparados con cada evidencia para determinar su relación. Se pudo observar que la evidencia 1.1., Caracteriza el proyecto de acuerdo con su índole o naturaleza social, económica, de inversión (entre otros) y el entorno utilizando referentes apropiados, está relacionada a los criterios de evaluación; CE3, CE4, CE5 y CE6. Por su parte, la evidencia 1.2., 
Aplica las metodologías apropiadas para formulación de un proyecto", se relaciona con los criterios de evaluación: CE1, CE2 y CE7. El primer resultado indica que: $58.3 \%$ de los criterios de evaluación de esta asignatura están relacionados al desempeño requerido para lograr buenos resultados en las pruebas de estado. En la figura 6 , se representa la aplicación del modelo, en sus cinco fases, con las entradas y las salidas. Estos resultados pueden hallarse de la cantidad de criterios que se alinean con las evidencias de la prueba Saber Pro sobre la cantidad de criterios totales de la asignatura.

Figura 6

Aplicación del Modelo de las cinco fases

\section{Micro Curriculo}

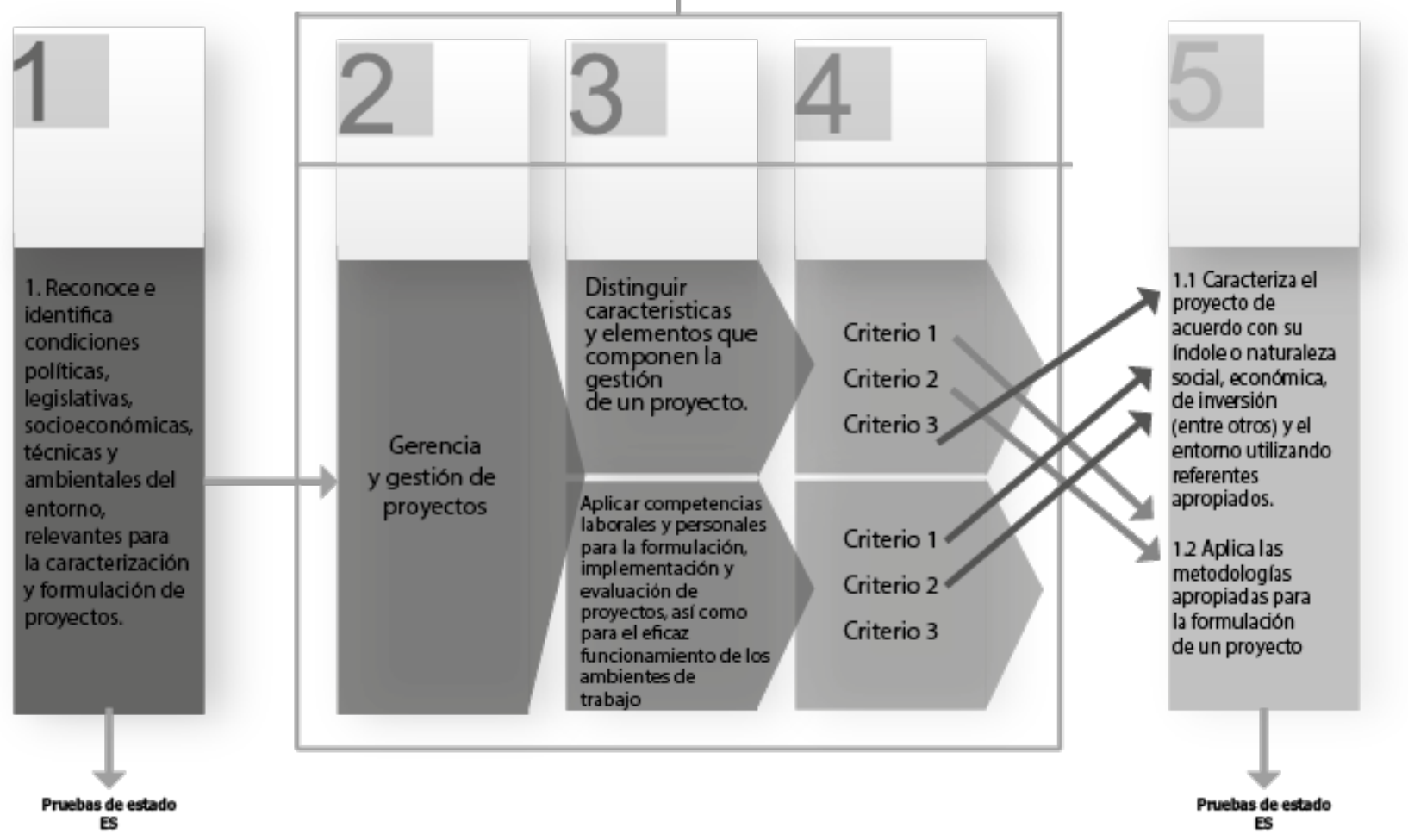

La aplicación del modelo se realizó con las otras asignaturas seleccionadas y se presentaron los siguientes resultados:

La afirmación 1 del módulo se relacionó con la asignatura Metodologías para el manejo de información y se tiene que el $42 \%$ de los criterios de evaluación se encuentran relacionados con el desempeño que mide la afirmación en cuestión. Esta asignatura potencia en lo relacionado a los procedimientos para la caracterización y formulación de un proyecto de ingeniería. Para Emprendimiento, se tiene que el 33,3\% de los criterios de evaluación, se relacionan con la afirmación y fortalece desde las competencias como generador de empresa. Economía para ingenieros y Teoría y modelos organizacionales, contribuyen con el $15 \%$ y $55 \%$ respectivamente en el desarrollo de competencias. Estos resultados permiten replantear las estrategias que se utilizan al interior del aula para seguir fortaleciendo las competencias mediante los desempeños que se asocian con las evidencias en la fase 5.

Una vez finalizada la aplicación del modelo de validación de las asignaturas seleccionadas para la afirmación número uno, se inicia con la afirmación número dos del módulo: "formula y evalúa el proyecto, apoyándose en un marco metodológico pertinente, a partir de las consideraciones del entorno y del análisis de alternativas". Esta afirmación abarca gran parte de las asignaturas propuestas para el segundo ciclo de la carrera de ingeniería de sistemas, sin embargo, para este ejercicio fueron seleccionadas todas aquellas asignaturas que se enfocan en 
la metodología, análisis y cuantificación de la información, que es aplicable para el desarrollo de un proyecto de ingeniería.

Al finalizar la aplicación del modelo de las 5 fases, articulado a un módulo específico, pueden resultar ciertas recomendaciones que impliquen, por ejemplo, tener en cuenta otras asignaturas que no se consideraron, pero que al final del análisis resultan claves en la estrategia o que hay fortalezas respecto al desempeño de algunas evidencias o por el contrario, se requiere plantear estrategias en el aula, para que estas evidencias ausentes o débiles en el micro currículo, sean desarrolladas convirtiéndola en fortaleza. El modelo permite la generación de estrategias que fortalezcan la implementación de las metodologías activas en el aula y la forma en que se evalúan los saberes específicos de los estudiantes teniendo en cuenta la medición de los desempeños.

\section{Conclusiones}

El aseguramiento de la calidad de la educación es un proceso que busca analizar la pertinencia curricular de una institución con el fin de garantizar la excelencia académica, un factor que invita a la mejora continua de las universidades desde los ejes operativos hasta los estratégicos, incentivando a que se adopte una cultura y filosofía de la evaluación institucional para medir; el sentido de ser de las IES y sus procesos académicos y de investigación enfocados a fortalecer su quehacer. En esa mejora continua, las pruebas estatales juegan un papel fundamental, debido a que permiten comprender la evolución del conocimiento de los estudiantes en su proceso de formación, convirtiéndose en un indicador relevante de medición para las instituciones; esto conlleva a implementar acciones académicas para la apropiación de estos procesos, sin embargo, se evidencian falencias en la manera como se abordan las operaciones para enfrentar al estudiante a dichas pruebas; estas operaciones generalmente se perciben desde los mecanismos de salida, que están enfocados solamente a los resultados del proceso evaluativo, como por ejemplo simulacros o desarrollo de pruebas similares a las estatales, minimizando el sentido de ser del problema, en un simple entrenamiento, que en muchas ocasiones no da resultados.

Por lo tanto se hace necesario buscar mecanismos de mejora que propendan por intervenir la raíz del problema, que no solo radica en tener mejores resultados en las pruebas estatales, sino en que las instituciones sean capaces de formar al estudiante para enfrentarse a diferentes técnicas evaluativas, producto de esta necesidad se plantea el modelo de 5 fases, que propende por entender la evaluación, como un proceso holístico que presenta unas entradas, las cuales se transforman para adquirir un resultado, que para el modelo se denomina salida, este método está estructurado en 3 niveles, eficiencia, eficacia y efectividad, el cual contrasta el proceso de planeación (¿qué se va a hacer?) con el operacional (lo que realmente se implementa), que para el caso de estudio, planea alcanzar la excelencia académica, por lo tanto se debe trabajar desde la generación de estrategias de evaluación y metodologías activas.

Este modelo de las 5 fases se encuentra en el primer nivel de implementación, el nivel de eficiencia, que tiene como propósito plantear acciones desde la universidad para fortalecer las pruebas estatales, para lo cual se realiza un primer ejercicio evaluativo, desde un estudio de caso, el programa de Ingeniería de Sistemas de Unipanamericana. Para aplicar el modelo se analizan las mallas curriculares del programa, se comprenden los módulos genéricos de las pruebas estatales, en específico las Saber Pro y se valida la malla del programa con respecto a las competencias que evalúa las pruebas, con el propósito de identificar la pertinencia del currículo, y fortalecer el proceso evaluativo en la institución. Sin embargo este es apenas el primer paso que se emprende, encaminado en lograr el objetivo de alcanzar la excelencia académica, quedan discusiones en torno a; ¿qué acciones implementar desde el currículo para fortalecer los procesos de evaluación?, ¿cómo lograr una medición que garantice la implementación de acciones que aporten al desarrollo de las pruebas estatales?, ¿qué resultados genera la implementación del modelo en la fase de salida? y ¿cómo lograr mantener procesos de 
retroalimentación constantes que aporten a la mejora continua con el fin asegurar la calidad de las instituciones de educación superior?.

\section{Referencias bibliográficas}

ANECA. (2013). Guía de apoyo para la redacción, puesta en práctica y evaluación de los resultados del aprendizaje. Madrid, España. Recuperado de: https://www.educacion.to.uclm.es/pdf/Calidad/learningoutcomes_v02.pdf

Arango, C. y Pinzon W. (2014). Sistema de producción Toyota usando cibernética de tercer orden,» Épsilon, pp. 175-195, 2014.

Ashby, W. (2018). An introduction to cybernetics. Creative Media Partners. Sacramento.

Unesco (2010). Compendio de la Declaración Mundial sobre la Educación Superior. Recuperado de Unesco.org: http://www.unesco.org/education/educprog/wche/compendio.htm

Bravo, N. (2007). Competencias proyecto Tuning - Europa, Tuning - America Latina. Bogotá. Recuperado de: http://www.cca.org.mx/profesores/cursos/hmfbcp_ut/pdfs/m1/competencias_proyectotuning.pdf

Buckler, B. (1996), "A learning process model to achieve continuous improvement and innovation", The Learning Organization, Vol. 3 No. 3, pp. 31-39. Recuperado de https://doi.org/10.1108/09696479610119660

Bhuiyan, N. \& Baghel, A. (2005), "An overview of continuous improvement: from the past to the present", Management Decision, Vol. 43 No. 5, pp. 761-771. Recuperado de https://doi.org/10.1108/00251740510597761

CNA. (2019). Consejo Nacional de Acreditación. Recuperado de www.cna.gov.co

Comisión Europea. (2009). El Marco Europeo de Cualificaciones para el aprendizaje permanente (EQF-MEC). Luxemburgo: Oficina de Publicaciones Oficiales de las Comunidades Europeas. Recuperado de: https://ec.europa.eu/ploteus/sites/eac-eqf/files/broch_es.pdf

D'Azzo, J. J., \& Houpis, C. D. (1995). Linear control system analysis and design: conventional and modern. McGraw-Hill Higher Education. New York.

Dorf, R. C., \& Bishop, R. H. (2017). Modern control systems. Pearson. Londres.

Firgermann, H. (2014). La Guía. Recuperado de https://educacion.laguia2000.com/evaluacion/criterios-deevaluacion

Gupta, P. (2006). Beyond PDCA-A new process management model. Quality progress, 39(7), 45.

Huarng, F., Horng, C., \& Chen, C. (1999). A study of ISO 9000 process, motivation and performance. Total Quality Management, 10(7), 1009-1025.

Hurtado, J. (1998). Metodología de la investigación holística. Fundacite-SYPAL. Caracas, 1998.

ICFES. (2019). Memorias de taller uso de resultados examen Saber Pro. Bogotá. Repueperado de https://www.icfes.gov.co/documents/20143/1112530/Taller\%20Uso\%20de\%20resultados\%20saber\%20pr ०\%20-\%202018.pdf

Johnson, C. N. (2002). The benefits fo PDCA. Quality Progress, 35(5), 120. 
Kuo, B. C., \& Golnaraghi, F. (1995). Automatic control systems(Vol. 9). Englewood Cliffs, NJ: Prentice-Hall. London.

López, M. (2013). Aprendizaje, competencias y TIC. Pearson. México D.F.

Magisterio. (2016). Contribución del modelo de evidencias al diseño de evaluaciones estandarizadas y de aula Recuperado de https://www.magisterio.com.co/articulo/contribucion-del-modelo-de-evidencias-aldiseno-de-evaluaciones-estandarizadas-y-de-aula

Mariano, M. (3002). Filosofía del lenguaje, lógica, filosofía de la ciencia y metafísica. Vol. 1. Editorial MAD. España.

MEN (2019). Oferta de combinatorias de módulos para 2019-1 Saber TyT. Recuperado de: http://www.icfes.gov.co/documents/20143/1476633/Oferta+de+combinatorias+de+modulos+para-20191+tyt.pdf/1bb0db59-17fc-315c-f1f5-49aa5f754424

Moen, R., \& Norman, C. (2006). Evolution of the PDCA cycle. Recuperado de http://www.idemployee.id.tue.nl/g.w.m.rauterberg/lecturenotes/DG000\%20DRP-R/references/MoenNorman-2009.pdf

Nova, N., Pinzón, W. \& Quintero, R. (2013). Hacia un nuevo modelo de cibernética, una aproximación al tercer orden. Universidad Distrital Francisco José de Caldas

Nemiche, M. (2004). Un modelo sistémico de evolución social dual. Universitat de València.

Ogata, K. (2003). Ingeniería de control moderna. Pearson Educación. London.

Ogata, K. (1987). Dinámica de sistemas. Prentice-Hall Hispanoamericana. México.

Oullet, A. (2000). La evolución informativa al servicio de las competencias. Revista Escuela de administración de negocios. Bogotá.

Páramo, P. (2014). La Recolección de la Información en las Ciencias Sociales: Una Aproximación Integradora. Lemoide Editores. Bogotá.

Pimienta, J. (2012). Las competencias en la docencia universitaria. Pearson. Máxico D.F.

Real Academia Española. (2018). Diccionario de la real academia de la lengua española. Madrid, España. Recuperado de https://dle.rae.es/?id=AOfanvT|A0gTnnL.

Rocha, J., Arango, C. \& Pinzón, W. Designed as a teaching recreational using third order cybernetics. In Proceedings of the 13th Latin American and Caribbean Conference for Engineering and Technology: Engineering Education Facing the Grand Challenges, What Are We Doing? number 216.

Silva, J., Bernal, E., \& Hernández, C. (2014). Modelo de aseguramiento interno de la calidad para las instituciones de educación superior en el marco del mejoramiento continuo de la calidad de la educación superior en Colombia. Recuperado de https://www.cna.gov.co/1741/articles-

186502_Modelo_aseguramiento.pdf

Tobón, S. (2013). Formación integral y competencias: Pensamiento complejo, currículo, didáctica y evaluación. 4 Ed. Editorial. Ecoe Ediciones. Bogotá

Yolles, M. (2006). Organization as complex systems: An introduction to knowledge cybernetics, Liverpool: Editorial Edad, 2006.

$$
\begin{aligned}
& \text { Esta obra está bajo una Licencia Creative Commons } \\
& \text { Attribución-NoCommercial } 4.0 \text { International } \\
& \qquad(\text { cc) BY-NC }
\end{aligned}
$$

